\title{
Commentary
}

\section{New Insights into the Biology of the BDNF Transcriptional} 'Code'

\author{
Minseok Song' and Francis S Lee \\ 'Department of Psychiatry, Weill Cornell Medical College of Cornell University, New York, NY, USA; '2Sackler Institute for Developmental \\ Psychobiology, Weill Cornell Medical College of Cornell University, New York, NY, USA
}

Neuropsychopharmacology (2016) 4I, 194I-1942; doi:10.1038/npp.2016.29

Brain-derived neurotrophic factor (BDNF) is a member of the neurotrophin family shown initially to influence the differentiation and survival of neurons (Barde et al, 1982) and subsequently established to mediate in the central nervous system (CNS) higher order activities, such as synaptic plasticity, and learning and memory (Park and Poo, 2013). BDNF is widely expressed throughout the CNS, and in response to neuronal depolarization, BDNF is secreted into the synapse leading to the activation of tropomyosin receptor kinase B and its downstream signaling cascades that contribute to gene transcriptional events critical for synaptic plasticity and cognitive function. Interestingly, among the growth factors in the CNS, BDNF is one of the few that has its own expression regulated by neuronal activity.

BDNF expression, both in rodents and in humans, is under tight control by nine distinct promoters, as well as two $3^{\prime}$ UTR polyadenylation sites. The entire coding sequence is in exon 9 and each of the eight upstream exons is spliced to exon 9 (West et al, 2014). Combinatorial use of multiple promoters and polyadenylation sites generate at least 18 distinct BDNF transcripts; however, these different BDNF transcripts ultimately lead to translation of the same BDNF protein. This high level of transcriptional regulation has been postulated to generate a putative 'code' that allows for exquisite spatiotemporal and stimuli-dependent regulation of BDNF expression within different subdomains of the neurons, as well as in different brain regions.

In line with BDNF's multiple roles on neuronal functions, genetically modified loss-of-function BDNF mice display a variety of behavioral phenotypes ranging from the expected altered learning and memory to a series of disparate behaviors, including increased anxiety, hyperphagiainduced obesity, aggression, hyperactivity, and impaired response to antidepressant treatments (Lindholm and Castren, 2014). There have been continued attempts to understand the relationship between the diversity of mRNA

*Correspondence: Dr FS Lee, Sackler Institute for Developmental Psychobiology, Weill Cornell Medical College of Cornell University, I 300 York Avenue, New York, NY I0065, USA, Tel: + I 212746 5403, Fax: + | 212746 64|4, E-mail: fslee@med.cornell.edu transcripts and in vivo biological functions of BDNF. Previously, the role of BDNF promoter IV has received the most attention as it was shown to be critical for activitydependent BDNF expression in the cortex. Genetically altered mice with a targeted point mutation in the CREB binding site in $B d n f$ promoter IV disrupted the activitydependent component of BDNF transcription and led to deficient inhibitory synapse development in the cortex (Hong et al, 2008). Using an alternative approach, another promoter IV mutant knock-in mouse was generated in which a GFP-STOP cassette replaced promoter IV (Sakata et al, 2009). These mice displayed significant deficits in GABAergic transmission and plasticity, as well as altered the spatial memory reversal and contextual fear extinction. Notably, in these mouse lines, no alterations in aggression or feeding behaviors were observed. Analyses of both of these promoter IV specific BDNF mice provided initial insights on how loss of a discrete BDNF transcript could contribute to the development of specific neuronal circuit deficits, as well as defined behavioral abnormalities, but left open questions with regards to the in vivo impact of the other BDNF transcripts.

In this context, in this issue, a study by Maynard et al (2016) addresses this question through the elegant and comprehensive analyses of four newly generated BDNF mouse lines with selective loss of individual BDNF promoters utilizing the previously established knock-in strategy of replacing individual exons with a GFP-STOP cassette. They examined whether different transcripts are related to specific BDNF-dependent phenotypes with these four mouse lines (Bdnf-e1, -e2, -e4, and -e6 mice) in which BDNF production is selectively impaired from each of the four major promoters (I, II, IV, or VI). Of note, promoter I and IV are the two promoters that have previously been shown to be induced by neuronal activity (Park and Poo, 2013). Intriguingly, Bdnf-e1 and -e2 mice displayed significantly different molecular, cellular, and behavioral phenotypes from Bdnf-e4 and -e6 mice. First, the impact of each of the promoters on BDNF protein levels was complex, but, strikingly, in the prefrontal cortical and hippocampal regions Bdnf-e1 mice had little loss of BDNF protein levels as 
compared with those in Bdnf-e4 mice. Conversely, BDNF levels in the hypothalamus of Bdnf-e1 and -e2 mice were significantly lower as compared with wild-type mice. In addition, these mice segregated into two categories in which genes in serotonergic signaling (Bdnf-e1 and -e2 mice) or in GABAergic signaling (Bdnf-e4 and -e6 mice) have been altered.

These molecular differences corresponded to strikingly different behavioral phenotypes, notably with regard to aggression. Male Bdnf-e4 and -e6 mice displayed no alteration in social behavior related to aggressive behavior. In contrast, male Bdnf-e1 and -e2 mice displayed a profound increase in aggressive behavior that was even higher than that reported for previous global BDNF knockout mice. The simultaneous comparison of all four mouse lines in this behavioral domain provided one of the strongest evidence that the different $B d n f$ transcripts impact separate behavioral domains.

The differential expression of BDNF transcripts in these four mouse lines in the hypothalamus suggested that this region may play an important role in mediating this aggression phenotype in the Bdnf-e1 and -e2 mice. While the role of BDNF in the hypothalamus was previously examined using hypothalamic specific knockout mice and focused on differences in feeding behavior (Lindholm and Castren, 2014), this current study suggests that $B d n f$ promoter I and II may play key roles in aggressive behaviors within this region. In this context, recent studies have already implicated hypothalamic subregions in relation to aggression (Lin et al, 2011). Future studies utilizing the Bdnf-e1 and -e2 mice will be informative in identifying the impact of these transcripts on specific cell types within the hypothalamus. More broadly, this study by Maynard et al (2016) introduces a valuable set of BDNF-related tools to begin to systematically dissect the in vivo impact of the individual BDNF promoters on discrete neuronal populations as well as circuits. Such future investigations may provide insights into how so many behavioral alterations initially observed in the global BDNF knockout mice can be understood through the framework of the complex BDNF transcriptional 'code'. Finally, these series of mouse lines will also provide much needed in vivo model systems to begin to interpret the myriad rodent and human studies that have described alterations in multiple BDNF transcripts in different brain regions under normal and pathological conditions.

\section{FUNDING AND DISCLOSURE}

The authors declare no conflict of interest.

\section{REFERENCES}

Barde YA, Edgar D, Thoenen H (1982). Purification of a new neurotrophic factor from mammalian brain. EMBO J 1: 549-553.

Hong EJ, McCord AE, Greenberg ME (2008). A biological function for the neuronal activity-dependent component of Bdnf transcription in the development of cortical inhibition. Neuron 60: 610-624.

Lin D, Boyle MP, Dollar P, Lee H, Lein ES, Perona P et al (2011). Functional identification of an aggression locus in the mouse hypothalamus. Nature 470: 221-226.

Lindholm JS, Castren E (2014). Mice with altered BDNF signaling as models for mood disorders and antidepressant effects. Front Behav Neurosci 8: 143.

Maynard KR, Hill JL, Calcaterra NE, Palko ME, Kardian A, Paredes $\mathrm{D}$ et al (2016). Functional role of BDNF production from unique promoters in aggression and serotonin signaling. Neuropsychopharmacol (e-pub ahead of print).

Park H, Poo MM (2013). Neurotrophin regulation of neural circuit development and function. Nat Rev Neurosci 14: 7-23.

Sakata K, Woo NH, Martinowich K, Greene JS, Schloesser RJ, Shen $\mathrm{L}$ et al (2009). Critical role of promoter IV-driven BDNF transcription in GABAergic transmission and synaptic plasticity in the prefrontal cortex. Proc Natl Acad Sci USA 106: 5942-5947.

West AE, Pruunsild P, Timmusk T (2014). Neurotrophins: transcription and translation. Handb Exp Pharmacol 220: $67-100$. 